\title{
Unusual Case of Spontaneous Extrahepatic Bile Duct Perforation with Vague Clinical Presentation
}

\author{
Dr. Brajesh Kumar ${ }^{1}$, Dr. Vivek Bhasker², Dr. Bikram Kumar ${ }^{3}$ \\ ${ }^{1}$ JRA-II, General Surgery, RIMS, Ranchi, Jharkhand, India \\ ${ }^{2}$ Senior Resident, General Surgery, RIMS, Ranchi, Jharkhand, India \\ ${ }^{3}$ JRA-II, General Surgery, RIMS, Ranchi, Jharkhand, India
}

\begin{abstract}
Spontaneous extrahepatic bile duct perforation is a rare entity. In this case report we will see that diagnosis in such cases is very difficult. Patient in this case was managed by exploratory laparotomy, CBD exploration and CBD repair with T-tube insertion.
\end{abstract}

Keywords: CBD- Common Bile Duct, TLC- Total Leucocyte Count, choledocholithiasis, T-tube

\section{Introduction}

Spontaneous perforation of extrahepatic bile duct not associated with any primary pathology is very rare. In most of the reported cases some associated cause is present. Most common associated pathologies in infants are choledochal cysts and anomalous union of pancreaticobiliary ductal system (AUPBD). In adults most common associated pathologies are choledocholithiasis, choledochal cyst, previous CBD exploration, choledochoenterostomies, pregnancy and acalculous cholecystitis.

Here we report a case of spontaneous common bile duct (CBD) perforation. Clinical features were not very specific and the perforation was diagnosed only after exploratory laparotomy.

\section{Case Report}

18 years old female came to surgery OPD with complain of mild pain abdomen. On examination tenderness was present in epigastrium. General examination was normal and no jaundice was present. Patient already had an ultrasound report which showed diagnosis of cholelithiasis with dialated CBD and IHBRs. Patient was admitted and investigations were done. Reports showed total bilirubin- 1 $\mathrm{mg} / \mathrm{dl}$, TLC- $11.9 \times 10^{3} / \mathrm{ml}$, serum electrolytes (sodium, potassium and calcium) within normal limits. Conservative treatment was continued with analgesic, antibiotics and PPI etc. Patient was not improving and abdomen got distended 2 days after admission. Repeat ultrasound study was done which showed mild fluid collection in abdomen, cholelithiasis and dialated CBD. Blood examination reports showed total bilirubin $4.3 \mathrm{mg} / \mathrm{dl}$, direct bilirubin- $1.85 \mathrm{mg} / \mathrm{dl}$, prothrombin time- 18 seconds with INR- 1.30. On fourth day of admission decision of exploratory laparotomy was taken as the patient was not improving and abdominal distension and jaundice were increasing.

On abdominal exploration 2liters of bilious fluid was found in peritoneal cavity. There was a perforation at the junction of cystic duct with CBD. Multiple stones were felt both in gall bladder and CBD. Cholecystectomy was followed by CBD exploration. Multiple CBD stones were seen in the lumen. CBD stones were cleared and CBD closed over Ttube. Abdominal drain was put and abdomen was closed.
Post operatively the patient recovered well. Abdominal drain was removed after 48 hours. T-tube cholangiogrm was done on $10^{\text {th }}$ postoperative day which showed free flow of dye and no residual stone. T-tube was removed and patient was kept in observation for 1 more day. Patient was discharged in $12^{\text {th }}$ post-op day.
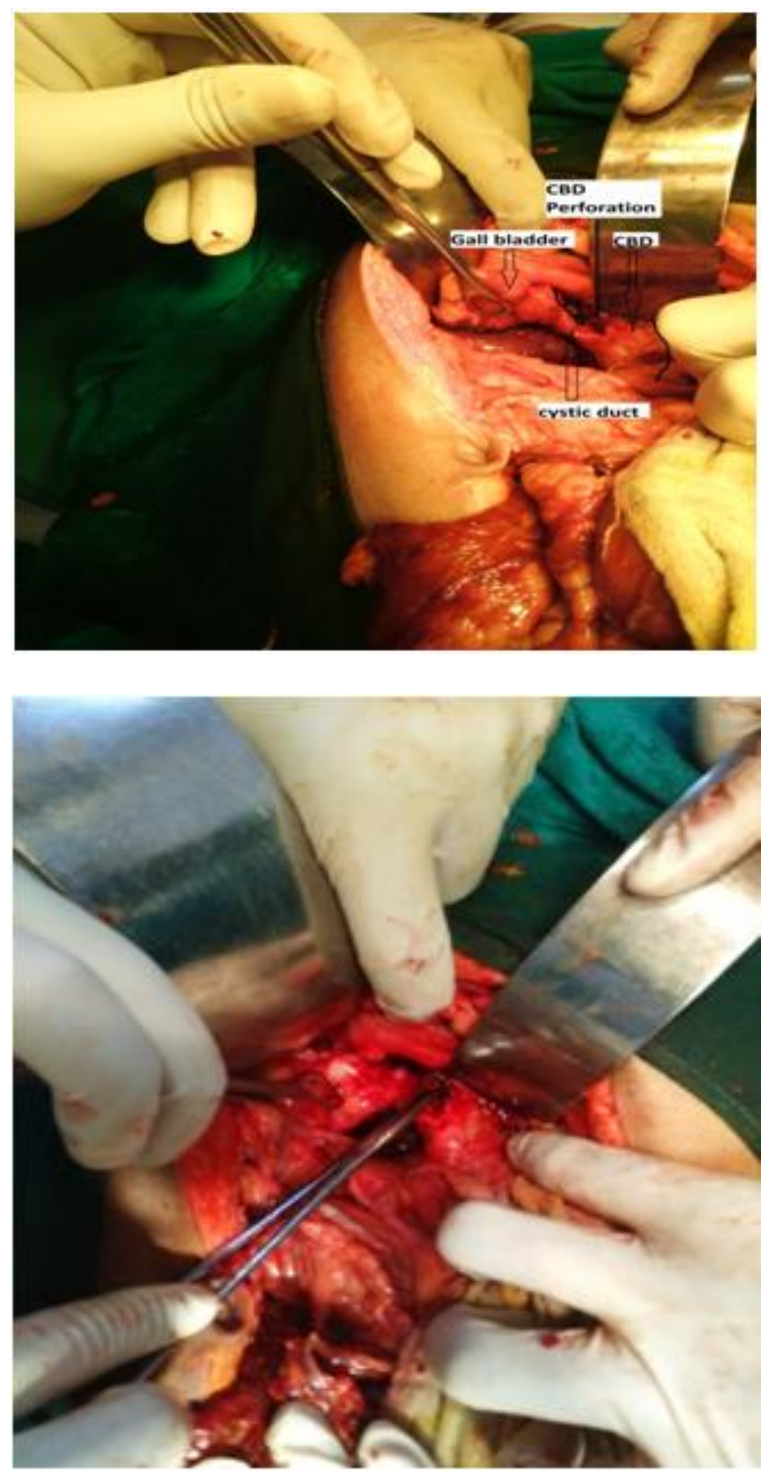

Figures showing Intraoperative findings 


\section{International Journal of Science and Research (IJSR) \\ ISSN (Online): 2319-7064}

Index Copernicus Value (2015): 78.96 | Impact Factor (2015): 6.391

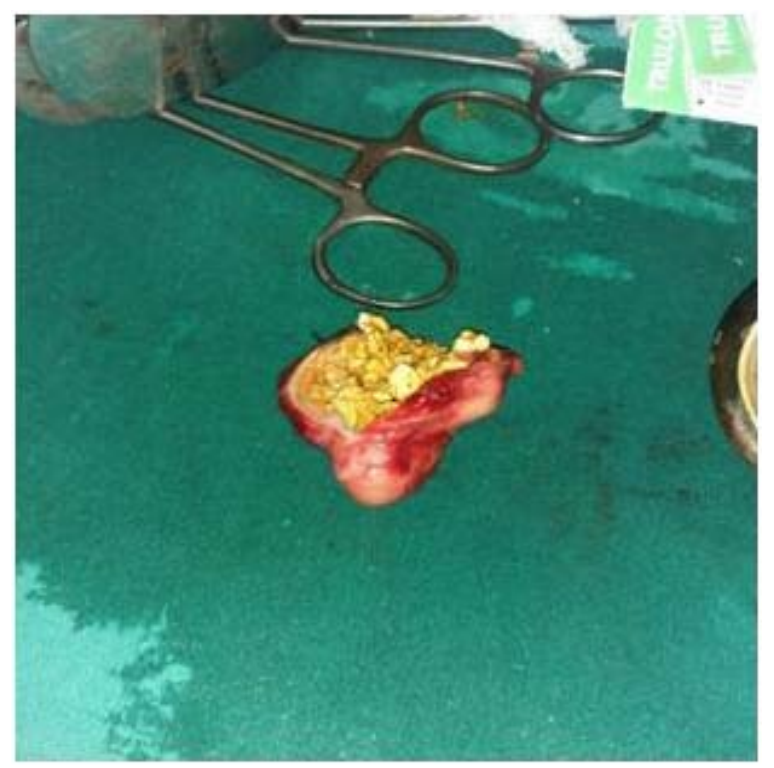

Figure showing gall bladder filled with stones

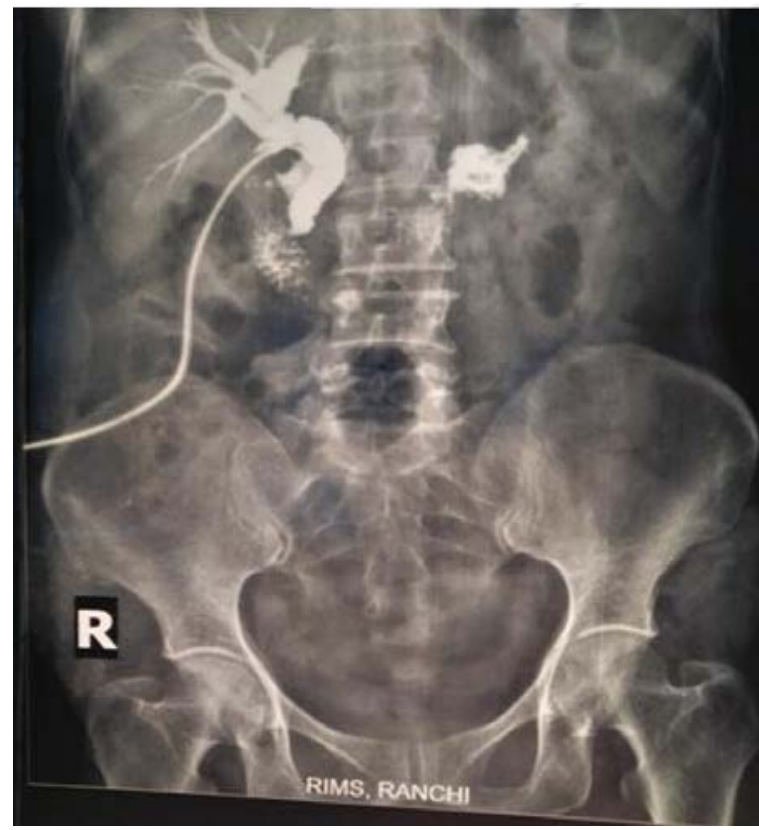

Figure showing free flow of dye in T-tube cholangiogram of the patient

\section{Discussion}

A spontaneous perforation of CBD is rare in adults [3] with perforation mostly found at the confluence of the cystic duct and common bile duct, which supports the suggestion of an area of developmental weakness [4]. Proposed theories for spontaneous CBD perforation include congenital weakness of the $\mathrm{CBD}$, distal obstruction and pancreatic reflux [1]. Most of the cases are associated with choledocholithiasis [5], other causes of spontaneous CBD perforation are choledochal cyst, site of previous CBD exploration, choledochoenterostomies, pregnancy, and acalculous cholecystitis [5]. Perforation of extra hepatic biliary tract is more common than intrahepatic biliary tract [1]. The presentation of CBD perforation may be acute or insidious, with insidious form being more common than acute form. Rarely, spontaneous CBD perforation may mimic gastric outlet obstruction. Duodenal perforation or gallbladder perforation.

The management depends on the type of presentation. In patients with insidious onset a staged management helps in converting emergency into an elective situation. In these patients percutaneous drainage of billiary collection or billiary decompression can be done initially for the billiary leak or collection followed by a definitive surgical repair. For patients with acute presentation the recommended treatment for common bile duct perforations is biliary decompression with $\mathrm{T}$ tube drainage of the common bile duct. In cases with distal obstruction of the CBD, a biliary enteric bypass can be done [1]. Primary suture repair of the common bile duct is considered unnecessary and even hazardous due to local inflammation and leak.

\section{Conclusion}

Spontaneous CBD perforation is rare. The diagnosis of CBD perforation in acute setting is challenging and it is important to have a high degree of suspicion especially in patients with presence of bile in the peritoneal cavity with normal stomach, duodenum and pancreas. Repair of perforation with T-tube insertion is safe and effective treatment in such cases.

\section{References}

[1] Marwah S, Sen J, Goyal A, Marwah N, Sharma JP. Spontaneous perforation of the common bile duct in an adult. Ann Saudi Med. 2005;25(1):58-59

[2] Lilly JR, Weintraub WH, Altman RP. Spontaneous perforation of the extrahepatic bile ducts and bile peritonitis in infancy. Surgery. 1974;75:664-673

[3] Kang SB, Han HS, Min SK, Lee HK, et al. Nontraumatic perforation of the bile duct in adults. Arch Surg. 2004; 139(10):1083-1087. doi: 10.1001/archsurg. 139.10.1083

[4] Steinhoff NG, Tucker GL. Nontraumatic perforation of the common duct. Am J Surg. 1971; 121(3):334-337. doi: 10.1016/0002-9610(71)90214-5.

[5] Rege SA, Lamba S, Sethi H. Spontaneous common bile duct perforation in adult: a case report and review. Int Surg. 2002;87:81-82

[6] Haller JO, Condon VR, Derdon WE. Spontaneous perforation of common bile duct in children. Radiology. 1989; 172:621-624

[7] Newell, E. D.: Spontaneous Rupture of the Common Bile Duct,Ann. Surg., 113:877, 1941.

[8] Reich, N. E.: Spontaneous Rupture of Normal Hepatic Duct, Ann. Surg., 116:137, 1942.

[9] Taube, H. N and Himel, H. A.: Non-Traumatic Perforation of Common Bile Duct, Am. J. Surg., 18:103, 1932.

[10] Vale, C. F. and Shapiro, H.: Non-Traumatic Perforation of Common Bile Duct, Am. J. Surg., 18:103, 1932.

[11]Verne, J. M.: Spontaneous Rupture of Hepatic Duct, Mem. Acad. Chir., 71:76, 1945 